\title{
Rehabilitative Care of Adult with Diabetes Mellitus
}

\author{
Habtamu Sewunet Mekonnen* \\ Department of Medical Nursing, University of Gondar, Ethiopia
}

Submission: October 24, 2018; Published: November 06, 2018

*Corresponding author: Habtamu Sewunet Mekonnen, Department of Medical Nursing, School of Nursing, College of Medicine and Health Sciences, University of Gondar, Gondar, Ethiopia

\begin{abstract}
Background: Diabetes of all types can lead to complications in many parts of the body and can increase the overall risk of dying prematurely. Possible complications include heart attack, stroke, kidney failure, leg amputation, vision loss and nerve damage. In pregnancy,
\end{abstract} poorly controlled diabetes increases the risk of fetal death and other complications

Aim: The main purpose of this study was to assess the rehabilitative care of a patient with diabetic mellitus

Result: diabetic patients need rehydration therapy, reversing electrolyte and acidosis, monitoring, blood pressure control, selfmanagement education, psychosocial intervention, and family and social support as rehabilitation.

Conclusion: The rehabilitation care should be given at all levels of health care delivery system to improve lifelong quality of diabetic patient, by preventing diabetic related burdens including social, economic and psychological impacts of the disease

Keywords: Diabetic mellitus; Rehabilitation; Hospitalization

Abbreviations: CAD: Coronary Artery Disease; DKA: Diabetic Ketoacidosis; DM: Diabetic Mellitus; HA1C: Gyrated Hemoglobin; IV: Intra Venous; NPH: Neutral Protamine Hagedorn; PH: Power of Hydrogen; SMBG: Self-Monitoring of Blood Glucose; UOG: University of Gondar; WHO: World Health Organization

\section{Background}

Diabetes is a group of metabolic diseases characterized by persistently high level of glucose in the blood that occurs when the body cannot produce enough insulin or cannot use insulin $[1,2]$. Type 2 diabetes is the one among the four clinical classes of diabetes, results from a progressive insulin secretory defect and peripheral tissue resistance to insulin. There may be some decrease in insulin production or a hyperinsulin state $[1,3]$.

Prevalence of both type 1 and type 2 diabetic mellitus is increasing worldwide including our setting, type 2 Diabetes is rising much more rapidly, presumably because of increasing obesity, reduced activity levels as countries become more industrialized, and the aging of the population $[4,5]$.

Diabetes of all types can lead to complications in many parts of the body and can increase the overall risk of dying prematurely. Possible complications include heart attack, stroke, kidney failure, leg amputation, vision loss and nerve damage. In pregnancy, poorly controlled diabetes increases the risk of fetal death and other complications $[1,6,7]$.

Developing a more intensive educational strategies on selfcare and medication adherence is key to achieving therapeutic goals in ambulatory care and reducing other potential risks, which leads to the development of diabetes complications [810].

In this essay the name of the patient was replaced by MJ for the sake of confidentiality, so I will use this name MJ throughout this essay.

\section{Patient Description}

MJ is a 47 years old female known hypertensive patient for the last 2 years. She is admitted to the Medical Ward, University of Gondar (UoG) Comprehensive Specialized Referral Hospital for the diagnosis of newly diagnosed type two Diabetes mellitus (DM) with diabetic ketoacidosis precipitated by pyelonephritis. She was referred from other health center and presented with a compliant of excess urination and excess drinking started two months back. As she reported, her urination amount and pattern was approximately 4-5 liters per day with the frequency of 1012 times per day and urge to drink water approximately 6-7 liters of water per day with the frequency of 8-10 times during the day time and 7-8 times during the night time associated with early satiety, dryness of lips and tongue and this is relieved after drinking water, shortness of breath associated with performing some activities, blurring of vision mostly during in the absence 
of light, intermittent headache on the right and left side of the head, tingling sensation and numbness usually on the right side of her arm, intermittent claudicating on her lower extremities during walking relieved by rest, bilateral flank pain, burning sensation during urination, one episode of projectile vomiting of ingested matter on admission, high grade intermittent fever with night sweating and fatigue, also she has epigasteric pain with bleaching and bloating of the abdomen after she takes meals prepared by berbere.

She has no history of delivery of a baby more than $4 \mathrm{~kg}$ and the presented clinical features described above. She has no personal habits of such as alcohol consumption, cigarette smoking and khat chewing. She had no family history of diabetic and cardiac disease, but she had family history of hypertension (her father).

\section{Discussion}

Diabetes is becoming a serious condition in the world, with increased need for health care. The World Health Organization (WHO) estimates that diabetes mellitus affects at least 285 million people and causes 3.2 million deaths, six deaths every minute and 8700 deaths every day, and this figure will increase by $70 \%$ in developed countries, and by $42 \%$ in developing countries by $2030[1,10,11]$.

The burden of diabetes has an impact not only on the quality of life of affected individuals and their families, but also on the country's socioeconomic structure because of in low and middle income countries, $29 \%$ of diabetes deaths occurs among people under the age of 50 , compared to $13 \%$ in high income countries which are the active work forces [11-13].The main goal in diabetes care is good quality of life, good metabolic control and minimization of complications caused by diabetes. Diabetes is a life-long challenge that needs behavioral change and adequate self-care practices to keep the illness under control $[1,2,4,14]$.

\section{Management}

Management of diabetes focuses on relieving of symptoms, prevention of acute and chronic complications, and Ensure weight reduction in overweight and obese individuals, maintain appropriate glycemic targets by applying best available interventions and follow up care $[2,4,6]$.

\section{Rehydration}

\section{Fluid replacement}

According to diabetic management guidelines, initial fluid therapy in diabetic ketoacidosis (DKA) directed toward expansion of the intravascular volume and restoration of renal perfusion [2]. Adequate rehydration with subsequent correction of the hyperosmolar state may result in a more robust response to low dose insulin therapy $[15,16]$. So, MJ was treated with Initial fluid therapy (with a loading dose of two litters normal saline and then the maintenance dose was followed) to replete the extracellular fluid volume without inducing cerebral edema due to rapid reduction in the plasma osmolality.

\section{Restoring electrolyte}

\section{Potassium supplementation}

Almost all patients with DKA have a considerable potassium deficit due to urinary and gastrointestinal losses. Most experts recommended potassium repletion after an initial infusion of isotonic saline to increase insulin responsiveness by lowering the plasma osmolality and if serum potassium concentration falls below $5.3 \mathrm{meq} / \mathrm{L}[2,16]$.

\section{Reversing acidosis}

\section{Insulin therapy}

Despite the standard's diabetic management guidelines, Insulin therapy lowers the serum glucose concentration by decreasing hepatic glucose production rather than enhancing peripheral utilization, diminishes ketone production by reducing both lipolysis and glucagon secretion. As a result, any dose of insulin that corrects the hyperglycemia will also normalize ketone metabolism. A continuous intravenous infusion of regular insulin is the treatment of choice, unless the episode of DKA is uncomplicated and mild. After resolution of DKA, patients treated with regular insulin received subcutaneous NPH and regular insulin twice daily, whereas patients treated with IV Glulisine insulin received Glargine once daily and glulisine before meals $[2,15,16]$. So, MJ is currently on subcutaneous NPH and regular insulin therapy.

\section{Monitoring}

Monitoring and target A1C (Gyrated hemoglobin), aim to achieve normal or near normal glycaemia with an $\mathrm{A} 1 \mathrm{C}$ goal of $<7$ percent. More stringent goals (i.e., a normal A1C, $<6.5$ percent) can be considered in individual patients. Less stringent treatment goals (e.g., < 8 percent) may be appropriate for patients with a history of severe hypoglycemia, patients with limited life expectancies, older adults, and individuals with comorbid conditions [16].

According to the American diabetic association standards of medical care in diabetes, the A1C test should be performed using a method that is certified by the National Glycohemoglobin standardization program and standardized or traceable to the Diabetes control and complications trial reference assay. Diabetic HgbA1c is a good marker for glucose control, because the more glucose is circulating in the blood, the more hemoglobin will be glycated. However, once hemoglobin is glycated, it stays that way until the red blood cell dies. Red blood cells live an average of three to four months. That is why $\mathrm{A} 1 \mathrm{C}$ level indicates an average glucose over the last few months and as an example, A1C of 7.0\% means an average blood glucose level of $154 \mathrm{mg} / \mathrm{dl}$ ) [17]. At that moment MJ's A1C level was not done, but I discussed with the medical intern the benefit of monitoring the A1C level of MJ.

MJ's serum glucose has been measured every hour, while serum electrolytes, blood urea nitrogen, creatinine, osmolality, and venous $\mathrm{pH}$ also measured every two to four hours. 


\section{Blood pressure control}

Hypertension is a common problem in type 1 and especially in type 2 diabetes. The American Diabetes Association recommends measuring blood pressure at every routine diabetes visit [18]. Early and effective treatment of blood pressure is important, both to prevent cardiovascular disease and to minimize the rate of progression of diabetic nephropathy and retinopathy [16]. However, MJ is a known hypertensive patient on follow up and her current blood pressure is $120 / 70 \mathrm{mmhg}$.

\section{Non-pharmacologic and rehabilitation}

\section{Self-management education}

The national standards on diabetic's self-management and education recommend and support all peoples with diabetics should involve in diabetics self-management education and to enhance knowledge attitude as well practice towards for quality diabetic self-care [2].

For effective management of diabetes, patients must be actively involved in self-care. This requires performance of many complex self-care behaviors including dietary control, regular exercise and psychosocial coping skills, medication adherence, and self-monitoring of blood glucose (SMBG) $[1,6,19]$. Therefore, I was trying to address MJ through counseling on exercise, nutritional, behavioral and showing how to take the ordered medication without interruption.

\section{Diet}

A review done by American Diabetes Association on Dietary Carbohydrate (Amount and Type) in the prevention and management of diabetes recommended the intake of diets that contain carbohydrate with high fiber and low glycemic load in order to reduce weight, controlling risk of coronary artery disease (CAD) and other complications [20].

Similarly, a study supports foods that have low glycemic index is important in mild glycemic control in diabetic patient. This mechanism is beneficial as patient treated with pharmacologic agent and also prevents increment of blood glucose level after intake of foods [21]. So, MJ was advised to reduce sugar content diets (such as sweet fruit juices and honey) and to take proportional amount of calorie intake (such as intake of whole grains, vegetables, legumes, and dairy products) as well as the negative effect of smoking and drinking much alcohol.

\section{Exercise}

I advised MJ to perform regular exercises, that supported by a standard management guideline of diabetics, to perform about a minimum of 150 minute in a week that is 21 minute exercise per day mild to moderate aerobic physical far apart at least three days and no more than two respective days without exercise [18]. Additionally, Diabetic patient without any problem would be motivated to perform strenuous exercise in at least two times per week [22].

\section{Psychosocial intervention}

The global diabetic care and America diabetic association supports that psychosocial care should be associated with a collaborative, patient approach method and provided to all people with diabetes, with the goals of optimizing health outcomes and health-related quality of life. Psychosocial intervention is not limited to the illness it may incorporates total quality of life in diabetics including behavioral, mood, attitude, financial and emotional $[1,18]$.

Psychosocial intervention is so important in improving regimen of treatment adherence, glycemic control, psychological, social welling and quality of life [23]. So, MJ is reassured and advised how to cope with her current anxiety and the social discrimination she perceived.

\section{Family and social support}

Despite a systematic review on the role of family in interventions among adults with diabetes, family should be motivated on self-management up on blood glucose control and other complications [24]. MJ's family advised to support psychologically and other needs of MJ, because the family dimension needs to be included to prove the effective family interventions.

\section{Nursing Implication}

Giving holistic care is the priority role of nurses, like that of other chronic conditions rehabilitative care of diabetes demands addressing the physical, social, economic and psychological aspects of both the patients and the family. Because many scholars agreed up on addressing these aspects for chronically ill patients had greatest role in halting disease morbidity and mortality.

Developing nursing care plan on rehabilitative care of diabetes, to identifying patient needs and intervene based on the identified gaps should be our prior duty. So, intervention based on evidence, with their rationale of giving that care by incorporating patients/their family in decision making is the corner stone of rehabilitative nursing care.

\section{Conclusion and Recommendation}

Since Diabetes is a chronic condition, most of diabetic patients should have to improve their self-care practice and life style in order to control the disease process and prevent the complications.

The rehabilitation care should be given at all levels of health care delivery system to improve lifelong quality of diabetic patient, by preventing diabetic related burdens including social, economic and psychological impacts of the disease [25].

Especially in developing countries like Ethiopia, late diagnosis of disease, absence of care at primary health care settings, lack of individualized glucose monitoring equipment's 
and absence of efficient education schedules increase the burden of the disease and its complication.

\section{Declarations}

\section{Consent to participate}

The study participant was informed about the purpose, method, expected benefit, and risk of the study. She also informed about her full right not to participate or withdraw from the study at any time and deciding not to participate had no impact on their services. Informed consent was obtained from study participant and anonymity was employed to maintained confidentiality.

\section{Acknowledgement}

I would like to express my gratitude to the University of Gondar College Of Medicine and Health School of Nursing Department of Medical Nursing, for their Permission and the study participants for their valuable information.

\section{Author Information}

Lecturer in Department of Medical Nursing, School of Nursing College of Medicine and Health Science, University of Gondar, Ethiopia.

\section{References}

1. Roopesh (2017) WHO updates fact sheet on Diabetes. communitymedicine 4 asses.

2. (2014) Standard Treatment Guidelines for General Hospitals. ( $3^{\text {rd }}$ edn), Ethiopia.

3. WHO | Diabetes.

4. (2011) WHO. Obesity and Overweight factsheet from the WHO.

5. Nigatu T (2012) Epidemiology, complications and management of diabetes in Ethiopia: A systematic review. J Diabetes 4(2): 174-180.

6. Hussain A, Claussen B, Ramachandran A, Williams R (2007) Prevention of type 2 diabetes: A review. Diabetes Res Clin Pract 76(3): 317-326.

7. Fact Sheets, Resources \& Publications, Diabetes, CDC.

8. Abebe SM, Berhane Y, Worku A (2014) Barriers to diabetes medication adherence in North West Ethiopia. Springerplus 3(1): 195.

9. Amente T, Belachew T, Hailu E, Berhanu N (2014) Self-care practice and its predictors among adults with diabetes mellitus on follow up at nekemte hospital diabetic clinic, west ethiopia. World J Med Med Sci 2(3): 1-16.

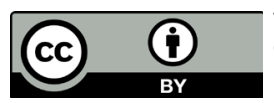

This work is licensed under Creative Commons Attribution 4.0 License DOI: 10.19080/JOJCS.2018.09.555758
10. Mehta RS, Karki P, Sharma SK (2006) Risk factors, associated health problems, reasons for admission and knowledge profile of diabetes patients admitted in BPKIHS. Kathmandu Univ Med J (KUMJ) 4(1): 1113.

11. Nijpels G Epidemiology of type 2 diabetes (revision number 18). Diapedia.

12. Whiting DR, Guariguata L, Weil C, Shaw J (2011) IDF Diabetes Atlas: Global estimates of the prevalence of diabetes for 2011 and 2030. Diabetes Res Clin Pract 94(3): 311-321.

13.Zimmet PZ, Alberti KG (2016) Epidemiology of Diabetes-Status of a Pandemic and Issues Around Metabolic Surgery. Diabetes Care 39(6): 878-883.

14. Saleh F, Mumu SJ, Ara F, Begum HA, Ali L (2012) Knowledge and selfcare practices regarding diabetes among newly diagnosed type 2 diabetics in Bangladesh: a cross-sectional study. BMC Public Health 12: 1112.

15. (2017) Standards of Medical Care In Diabetes.

16. Abbas E, Burton D Rose (2013) Treatment of diabetic ketoacidosis and hyperosmolar hyperglycemic state in adults.

17. American Diabetes Association (2014) Standards of Medical Care in Diabetes. Volume 37.

18. American Diabetes Association ${ }^{\circledR}$ Releases (2016) 2017 Standards of Medical Care in Diabetes: American Diabetes Association ${ }^{\circledR}$.

19. Lin K, Yang X, Yin G, Lin S (2016) Diabetes Self-Care Activities and Health-Related Quality-of-Life of individuals with Type 1 Diabetes Mellitus in Shantou, China. J Int Med Res 44(1): 147-156.

20. Sheard NF, Clark NG, Brand-Miller JC, Franz MJ, Pi-Sunyer FX, et al. (2004) Dietary carbohydrate (amount and type) in the prevention and management of diabetes: a statement by the american diabetes association. Diabetes Care 27(9): 2266-2271.

21. Nabi G, Azad KAK, Rahman NW, Rabbani R, Chowdhury TI (2017) Life Style Modification Among Diabetic Patients. J Dhaka Med Coll 25(1): 46-52.

22.Zanuso S, Jimenez A, Pugliese G, Corigliano G, Balducci S (2010) Exercise for the management of type 2 diabetes: a review of the evidence. Acta Diabetol 47(1): 15-22.

23. Redeker NS (1988) Health beliefs and adherence in chronic illness. J Nurs Scholarsh 20(1): 31-35.

24. Torenholt R, Schwennesen N, Willaing I (2014) Lost in translation-- the role of family in interventions among adults with diabetes: a systematic review. Diabet Med 31(1):15-23.

25. Steinsbekk A, Rygg LØ, Lisulo M, Rise MB, Fretheim A (2012) Group based diabetes self-management education compared to routine treatment for people with type 2 diabetes mellitus. A systematic review with meta-analysis. BMC Health Serv Res 12: 213.

\begin{tabular}{|l|}
\hline \multicolumn{1}{|c|}{ 'our next submission with Juniper Publishers you the below assets } \\
- Quality Editorial service \\
- Swift Peer Review \\
- Reprints availability \\
- E-prints Service \\
- Manuscript Podcast for convenient understanding \\
- Global attainment for your research \\
- Manuscript accessibility in different formats \\
( Pdf, E-pub, Full Text, Audio) \\
- Unceasing customer service \\
Track the below URL for one-step submission \\
https://juniperpublishers.com/online-submission.php \\
\hline
\end{tabular}

